\title{
Preventive catheter ablation in patients with post-infarct cardiomyopathy undergoing secondary prevention defibrillator implantation: A meta-analysis of randomized clinical trials
}

\author{
Babikir Kheiri ${ }^{1}$, Timothy Simpson ${ }^{1}$, and Babak Nazer ${ }^{1}$ \\ ${ }^{1}$ Oregon Health \& Science University
}

July 29,2020

\begin{abstract}
Introduction: In patients with post-infarct cardiomyopathy and ventricular arrhythmias (VT) necessitating implantable cardioverter defibrillators (ICD) are at risk of recurrent shocks with increased morbidity. Methods and Results: A comprehensive search of electronic databases for all randomized clinical trials that evaluated the role of catheter ablation as a preventive strategy at the time of secondary prevention ICD implantation was performed. Four trials were identified with a total of 505 patients (average age $66.4 \pm 9.0 \mathrm{yr} ; 87.7 \%$ were male). Preventive ablation was associated with a significant reduction in appropriate device therapies (shocks and/or anti-tachycardia therapy) (hazard ratio $[\mathrm{HR}]=0.62 ; 95 \%$ confidence interval $[\mathrm{CI}]=0.46-0.82$; $\mathrm{p}<0.01)$, sustained VT $(\mathrm{HR}=0.74 ; 95 \% \mathrm{CI}=0.55-0.99 ; \mathrm{p}=0.04)$ compared to control. There were no differences in inappropriate device shocks $(\mathrm{HR}=0.80 ; 95 \% \mathrm{CI}=0.38-1.71)$, all-cause death $(\mathrm{HR}=0.93 ; 95 \% \mathrm{CI}=0.53-1.64)$, cardiac death $(\mathrm{HR}=0.63 ; 95 \%$ $\mathrm{CI}=0.29-1.36)$, arrhythmic death $(\mathrm{HR}=0.26 ; 95 \% \mathrm{CI}=0.05-1.31)$, or cardiac hospitalization $(\mathrm{HR}=0.79 ; 95 \% \mathrm{CI}=0.57-1.11)$ between strategies. Preventive ablation was associated with improved SF-36 physical component (SMD=2.81; 95\% CI-0.53-5.10; $\mathrm{p}=0.02)$, but not the mental component $(\mathrm{SMD}=1.30 ; 95 \% \mathrm{CI}=-2.06-4.66)$. Conclusion: Among patients with post-infarct cardiomyopathy and VT, preventive catheter ablation at the time of ICD implantation is associated with a significant reduction of appropriate ICD therapy and sustained VT, and improvement in the physical component of quality-of-life, but no reduction in mortality.
\end{abstract}

\section{Introduction}

In patients with post-infarct cardiomyopathy who develop ventricular tachycardia (VT) or ventricular fibrillation (VF), implantable cardioverter defibrillators (ICDs) are an effective therapy to reduce the risk of sudden cardiac death. ${ }^{1}$ However, ICDs do not prevent recurrent arrhythmias and subsequent ICD discharges (shocks) are associated with increased morbidity and mortality, and significantly affect quality of life. Current guidelines recommend anti-arrhythmic drugs (AADs) as a first-line therapy for prevention of recurrent VT/VF leading to ICD shocks (Class I). In cases where AADs are ineffective, poorly tolerated, or contraindicated- catheter ablation is recommended (Class I). In patients in whom AADs are undesirable for other reasons, catheter ablation may be considered (Class IIa). ${ }^{1}$

Given the potential benefits of avoiding recurrent ICD discharges and chronic AAD side-effects, several randomized trials have evaluated the efficacy of preventative catheter ablation, but without consensus. Therefore, we conducted this meta-analysis to evaluate the role of catheter ablation as a preventive strategy at the time of secondary prevention ICD implantation in patients with post-infarct cardiomyopathy and VT.

\section{Methods}

This meta-analysis followed the Preferred Reporting Items for Systematic Reviews and Meta-Analyses (PRISMA) guidelines. We performed a comprehensive search of electronic databases for all randomized clinical 
trials (RCTs) from inception to March 2020 (BK and TS). The keywords used (VT OR ventricular tachycardia OR ventricular tachyarrhythmia) AND (catheter ablation OR radiofrequency ablation OR radiofrequency catheter ablation OR RFA OR ablation therapy). No language restriction was used. We excluded studies that were observational in design and/or not yet peer-reviewed and published. The risk of bias of the included trials was assessed using the Cochrane Collaboration tool.

The primary outcome was the frequency of appropriate device therapies defined as shocks or anti-tachycardia therapy. Secondary outcomes included appropriate device shocks, inappropriate device shock, sustained VT/VF, any death, cardiac death, arrhythmic death, and cardiac hospitalization during follow-up. Tertiary outcomes included quality-of-life and was assessed using the short-form 36 (SF-36) patient-reported outcome. We used a random-effects model to calculate hazard ratios (HRs) and standardized mean differences (SMD) with their corresponding 95\% confidence intervals (CIs) using an inverse variance method. Heterogeneity was evaluated using the $I^{2}$ statistic. Publication bias of the primary outcome was assessed using funnel plot analysis.

\section{Results}

We identified 4 RCTs ${ }^{2-5}$ which included 505 patients (246 preventive ablation, 259 control) with 10,353 patient-years of follow-up. Mean age was $66.4 \pm 9.0$ years and $87.7 \%$ were male. The average left ventricular ejection fraction was $35 \pm 9 \%$, and time from prior myocardial infarction to randomization was $10.1 \pm 9.0$ years. Only $25.9 \%$ of patients were on AADs (Table 1 ).

Analysis found preventive ablation was associated with a reduced frequency of appropriate device therapies $(\mathrm{HR}=0.62 ; 95 \% \mathrm{CI}=0.46-0.82 ; \mathrm{p}<0.01 ;$ number-needed-to-treat $[\mathrm{NNT}]=7)$, driven largely by a significant reduction of appropriate ICD shocks $(\mathrm{HR}=0.52 ; 95 \% \mathrm{CI}=0.36-0.75 ; \mathrm{p}<0.01 ; \mathrm{NNT}=8)$ compared with the control arm. The preventive ablation strategy was also associated with a significant reduction in frequency of sustained VT/VF $(\mathrm{HR}=0.74 ; 95 \% \mathrm{CI}=0.55-0.99 ; \mathrm{p}=0.04 ; \mathrm{NNT}=11)$ compared to control. There were no differences in inappropriate device shocks $(\mathrm{HR}=0.80 ; 95 \% \mathrm{CI}=0.38-1.71)$, any death $(\mathrm{HR}=0.93 ; 95 \% \mathrm{CI}=0.53-$ $1.64)$, cardiac death $(\mathrm{HR}=0.63 ; 95 \% \mathrm{CI}=0.29-1.36)$, arrhythmic death $(\mathrm{HR}=0.26 ; 95 \% \mathrm{CI}=0.05-1.31)$, or cardiac hospitalization ( $\mathrm{HR}=0.79 ; 95 \% \mathrm{CI}=0.57-1.11$ ) between strategies (Figure 1 ). QOL was measured in 3 RCTs ${ }^{2-4}$ Preventive ablation was associated with improved SF-36 physical component (SMD $=2.81 ; 95 \%$ CI-0.53-5.10; $\mathrm{p}=0.02)$, but scores were similar in the mental component ( $\mathrm{SMD}=1.30 ; 95 \% \mathrm{CI}=-2.06-4.66$; $\mathrm{p}=0.45)($ Figure 2 $)$.

There were 12 major complications (4.9\% of patients) in the ablation group: tamponade in $3(1.2 \%)$, major bleeding in $3(1.2 \%)$, third-degree atrioventricular block in $3(1.2 \%)$, stroke in $2(0.8 \%)$ and cardiac perforation requiring surgical repair in 1 patient $(0.4 \%)$.

\section{Discussion}

In this meta-analysis of RTCs evaluating preventative catheter ablation at time of ICD implantation for secondary prevention of VT/VF due to post-infarct cardiomyopathy, we found a significant reduction in appropriate device therapy and sustained VT/VF in patients undergoing preventative catheter ablation compared to routine care. In addition, ablation was associated with a significant improvement in the physical component of quality-of-life measures. Major complications in the ablation group were rare and there was no procedure-related mortality, indicating this strategy may have an acceptable risk profile.

Previous studies have demonstrated a significant reduction of VT recurrence with either amiodarone or catheter ablation among unselected patients (post-infarct and non-ischemic cardiomyopathy) with secondary prevention ICDs. ${ }^{6}$ However, most trials utilized catheter ablation only after multiple VT recurrences. In our investigation, the degree of reduction in appropriate ICD therapy was clinically meaningful, requiring only 7 patients undergo preemptive ablation for 1 to avoid ICD therapy. This degree of reduction, coupled with acceptable procedural safety and the benefit of avoiding the risks of chronic AADs, may confer clinically meaningful benefit. Moreover, preventative ablation produced meaningful improvements in quality-of-life, and may alleviate the debilitating effects of appropriate ICD shocks in these vulnerable patients. 
Important differences between included studies exist, particularly with regard to inclusion criteria: SMS and SMASH-VT enrolled patients with unstable VT or VF, VTACH enrolled stable VT, and BERLIN VT enrolled both stable and unstable VT. Importantly, significant cross-over from the ICD alone arms to the preventive ablation arms were observed (ranging 2-22\%). The first of these studies began enrollment in the year $2000,{ }^{5}$ and the most recent one conducted enrollment in $2018 .^{2}$ Thus, significant heterogeneity likely exists in mapping and ablation methods and technologies available to the investigators over this 18-year span. Furthermore, contemporary trends and guidelines likely influenced programming ICDs at higher rates and longer detection delays the more recent trials compared with the older ones.

Limitation of this study includes the relatively low number of enrolled patients and finite number of endpoints in analyzed trials. The lack of patient-level data prevents analysis of the influence of various mapping techniques, ablation strategies, device programming, and baseline AADs on the effects of preventive ablation. We hope the currently enrolling trials (PREVENTIVE VT [NCT03421834] and PAUSE-SCD [NCT02848781]) will provide further insights.

\section{Conclusion}

This meta-analysis demonstrates that in patients with post-infarct cardiomyopathy and VT, preventive catheter ablation at the time of ICD implantation is associated with a significantly reduced frequency of appropriate ICD therapy and sustained VT/VF, and improvement in the physical component of QOL, but no reduction in mortality. The NNT of 8 to spare one patient from ICD shock(s) should be weighed against a number-needed-to-harm of 21 based on a $4.9 \%$ incidence of major ablation-related complications. Further studies should address which subsets of patients will derive maximal net benefit from a preventive ablation strategy at time of secondary prevention ICD implant.

\section{References:}

1. Cronin EM, Bogun FM, Maury P, Peichl P, Chen M, Namboodiri N, Aguinaga L, Leite LR, Al-Khatib SM, Anter E, Berruezo A, Callans DJ, Chung MK, Cuculich P, d'Avila A, Deal BJ, Della Bella P, Deneke T, Dickfeld TM, Hadid C, Haqqani HM, Kay GN, Latchamsetty R, Marchlinski F, Miller JM, Nogami A, Patel AR, Pathak RK, Saenz Morales LC, Santangeli P, Sapp JL, Sarkozy A, Soejima K, Stevenson WG, Tedrow UB, Tzou WS, Varma N, Zeppenfeld K. 2019 HRS/EHRA/APHRS/LAHRS expert consensus statement on catheter ablation of ventricular arrhythmias: Executive summary. Hear Rhythm [Internet]. 2020;17:e155-e205. Available from: http://dx.doi.org/10.1016/j.hrthm.2019.03.014

2. Willems S, Tilz RR, Steven D, Kääb S, Wegscheider K, Geller L, Meyer C, Heeger C-H, Metzner A, Sinner MF, Schlüter M, Nordbeck P, Eckardt L, Bogossian H, Sultan A, Wenzel B, Kuck K-H. Preventive or Deferred Ablation of Ventricular Tachycardia in Patients with Ischemic Cardiomyopathy and Implantable Defibrillator (BERLIN VT): A Multicenter Randomized Trial. Circulation . 2020;141:1057-1067.

3. Kuck KH, Tilz RR, Deneke T, Hoffmann BA, Ventura R, Hansen PS, Zarse M, Hohnloser SH, Kautzner J, Willems S. Impact of substrate modification by catheter ablation on implantable cardioverter-defibrillator interventions in patients with unstable ventricular arrhythmias and coronary artery disease: Results from the multicenter randomized controlled SMS (Substrate . Circ Arrhythm Electrophysiol . 2017;10:1-9.

4. Kuck K, Schaumann A, Eckardt L, Willems S, Ventura R, Delacrétaz E, Pitschner H, Kautzner J, Schumacher B, Hansen P. Catheter ablation of stable ventricular tachycardia before defibrillator implantation in patients with coronary heart disease $(\mathrm{VTACH})$ : a multicentre randomised controlled trial. Lancet . 2010;375:31-40.

5. Reddy VY, Reynolds MR, Neuzil P, Richardson AW, Taborsky M, Jongnarangsin K, Kralovec S, Sediva L, Ruskin JN, Josephson ME. Prophylactic catheter ablation for the prevention of defibrillator therapy. $N$ Engl J Med . 2007;357:2657-2665.

6. Kheiri B, Barbarawi M, Zayed Y, Hicks M, Osman M, Rashdan L, Kyi HH, Bachuwa G, Hassan M, Stecker EC, Nazer B, Bhatt DL. Antiarrhythmic Drugs or Catheter Ablation in the Management of Ventricular 
Tachyarrhythmias in Patients with Implantable Cardioverter-Defibrillators: A Systematic Review and MetaAnalysis of Randomized Controlled Trials. Circ Arrhythmia Electrophysiol . 2019;12:1-10.

\section{Figures legend}

Figure 1: Forest plot of clinical outcomes.

Abbreviations: BERLIN VT: Preventive Ablation of Ventricular Tachycardia in Patients With Myocardial Infarction; SMASH-VT: Substrate Mapping and Ablation in Sinus Rhythm to Halt Ventricular Tachycardia study; SMS: Substrate Modification Study; VTACH: Ventricular Tachycardia Ablation in Coronary Heart Disease study.

Figure 2: Forest plot of quality-of-life outcomes.

Abbreviations: same as figure 1.
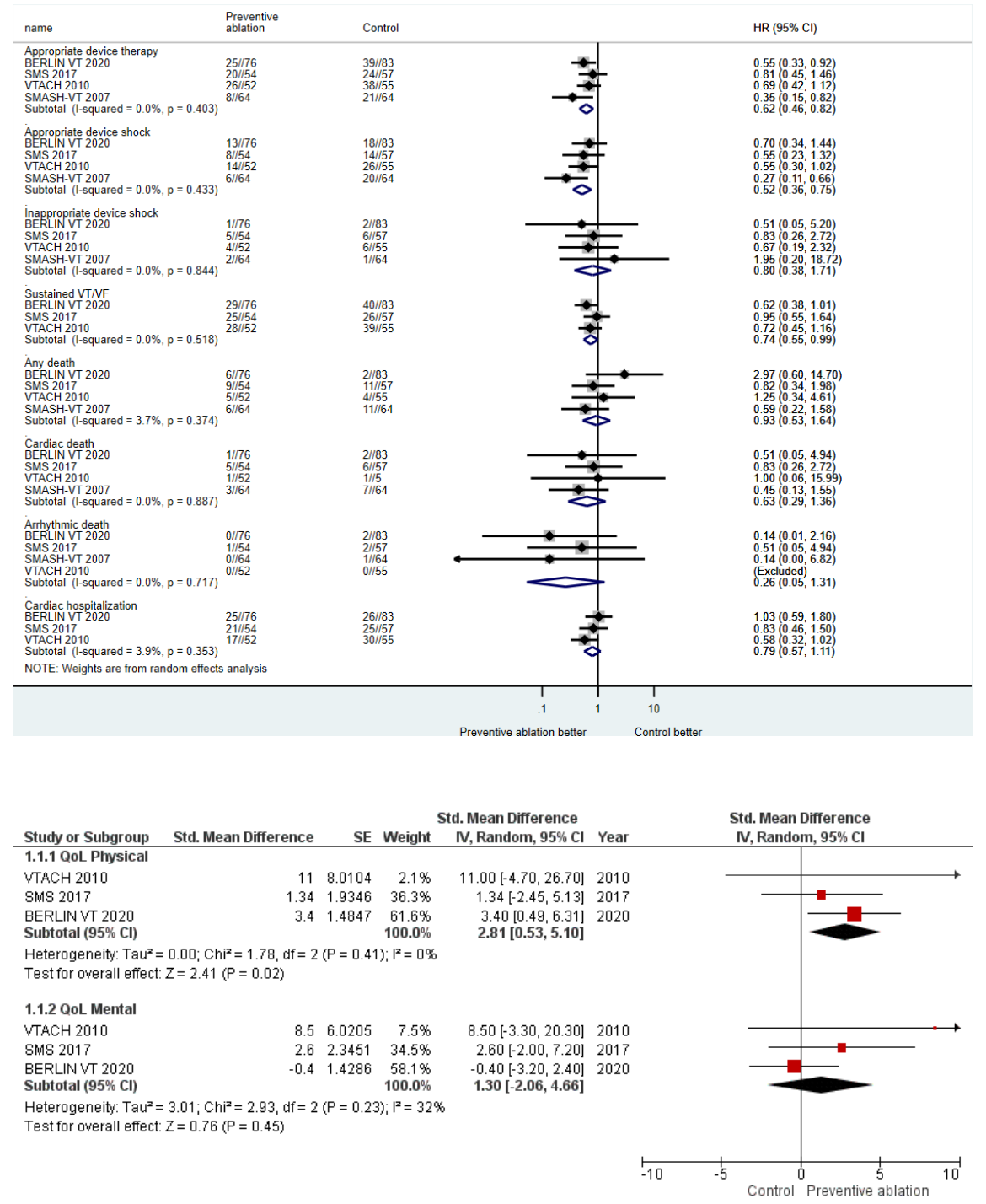

\section{Hosted file}


Table 1.docx available at https://authorea.com/users/347310/articles/473048-preventivecatheter-ablation-in-patients-with-post-infarct-cardiomyopathy-undergoing-secondaryprevention-defibrillator-implantation-a-meta-analysis-of-randomized-clinical-trials 